\title{
FUNCTIONAL MODEL TO ESTIMATE THE INELASTIC DISPLACEMENT RATIO
}

\author{
Vlad CEANGU - PhD Candidate, Technical University of Civil Engineering, e-mail: vlad.ceangu@outlook.com \\ Dan CRETU - Professor, PhD, Technical University of Civil Engineering, e-mail: cretud@utcb.ro
}

\begin{abstract}
In this paper a functional model to estimate the inelastic displacement ratio as a function of the ductility factor is presented. The coefficients of the functional model are approximated using nonlinear regression. The used data is in the form of computed displacement for an inelastic single degree of freedom system with a fixed ductility factor. The inelastic seismic response spectra of constant ductility factors are used for generating data. A method for selecting ground-motions that have similar frequency content to that of the ones picked for the comparison is presented. The variability of the seismic response of nonlinear single degree of freedom systems with different hysteretic behavior is presented.
\end{abstract}

Keywords: inelastic response spectra, ground-motion selection, nonlinear regression

\section{Introduction}

The inelastic displacement ratio is a response parameter that is often used in the design or assessment of buildings in seismic regions. This factor represents the ratio between the inelastic and elastic displacements of a single degree of freedom system with period $T_{i}$. Usually the structural engineer uses this ratio to multiply the elastic displacements of structures with a fixed strength factor. Empirical relations, or functional forms, are used to estimate the inelastic displacement ratio. In this paper a functional form is used, having as dependent variables the elastic period of the single degree of freedom $T_{i}$, the ground fundamental period at the site $T_{p}$ and a constant ductility factor $\mu_{k}$.

The response is not dependent on these three terms only. The advantage of these models is that using simple relations, with a few modeling parameters, the response is estimated with a particular confidence factor.

For the development of functional forms, inelastic seismic response spectra of constant ductility are estimated using recorded accelerograms. A method of selection of ground motions based on the shape of the elastic response spectra, the dissipated hysteretic energy spectra and the ground fundamental period at the site $T_{p}$ is used. Using this method the ground motions are not modified. The inelastic response spectra are computed using the algorithms presented in $[5,6]$.

The coefficients for the parameters of the functional model are approximated using the nonlinear regression method [3]. The uncertainty of the response is quantified using a functional form for the standard deviation observed for the data. The functional form in the regression model is composed of two parts. The first is a deterministic one and the second a stochastic one. The first term is considered as the estimate of the mean of the response and the second as the standard deviation of the response.

\section{Seismic Action}

The general criterion for selecting accelerograms is that the provided intensity measure $I M$ guarantee efficiency and sufficiency $[14,15]$. The efficiency and sufficiency of an intensity 
measure is expressed as a function of the response of a structural system to the chosen IM . A set of accelerograms is considered efficient if the standard deviation of the response, or engineering demand parameters EDP, have low values compared to other sets. A set of accelerograms is considered sufficient if the obtained engineering demand parameters are independent of the characteristics of the earthquake forecast model. For example, the EDP should be independent of the magnitude, distance and other parameters of the source of the seismic event. The duration of the event and the frequency content of the ground motion are path dependent, although the corner period is a function of the magnitude of the event [13].

The site of interest is situated in the Vrancea seismic zone, characterized by an elastic response spectrum with a corner period of $1.6 \mathrm{~s}$, as in the present seismic design code used in Romania [11]. The value of a particular intensity measure for single degree of systems is not of interest, so that the value of the peak ground acceleration is not considered.

The ground motion selection method, GMSM, is composed of two steps. The first step is to choose a ground motion from available data-bases of accelerograms that have the shape of the elastic response spectrum similar to the one in the design code. Two data-bases were used, the first one is PEER data-base [1] and the second is ROMPLUS data-base. A total of 26 accelerograms were selected, with 23 from the first data-base and 3 from the second one. The second step is the final selection of accelerograms, based on the shape of the hysteretic energy response spectra and the number of plastic cycles [6]. The shape of compared spectra is that for the 3 accelerograms selected as being those recorded in the site of interest. This way, a bias in the response is expected. Taking as criterion of comparison the shape of the spectra of these 3 accelerograms, the expected events will have general characteristics similar to the selected recorded ground motions [7]. In this step, ground-motions that have similar frequency content are selected. A final number of 15 real accelerograms is chosen. The advantage of this method of selection is that the scaling of accelerograms is avoided. Its disadvantage is that only a few scenarios of events are modeled and these are similar to the ones picked for the comparison. The list of the used accelerograms is found in the annex attached at the end of this paper.

In figure 1 the elastic response spectra for the absolute accelerations and relative displacements are plotted, together with the mean and the standard deviation. It is observed that the ground fundamental period $T_{p}$, defined as the period at which the maximum pseudo-velocity is computed, has values on all period intervals for each of the accelerograms.

Like any other intensity measure, an accelerogram must represent the seismic hazard for a specific probability of exceedance for the chosen site. In the ground-motion selection method, the probability of exceedance is assigned only for a scalar parameter $I M$, usually $P G A$ or $S_{a}(T)$. All the other parameters that have an influence on the vector of intensity measure $I M$ are not considered.
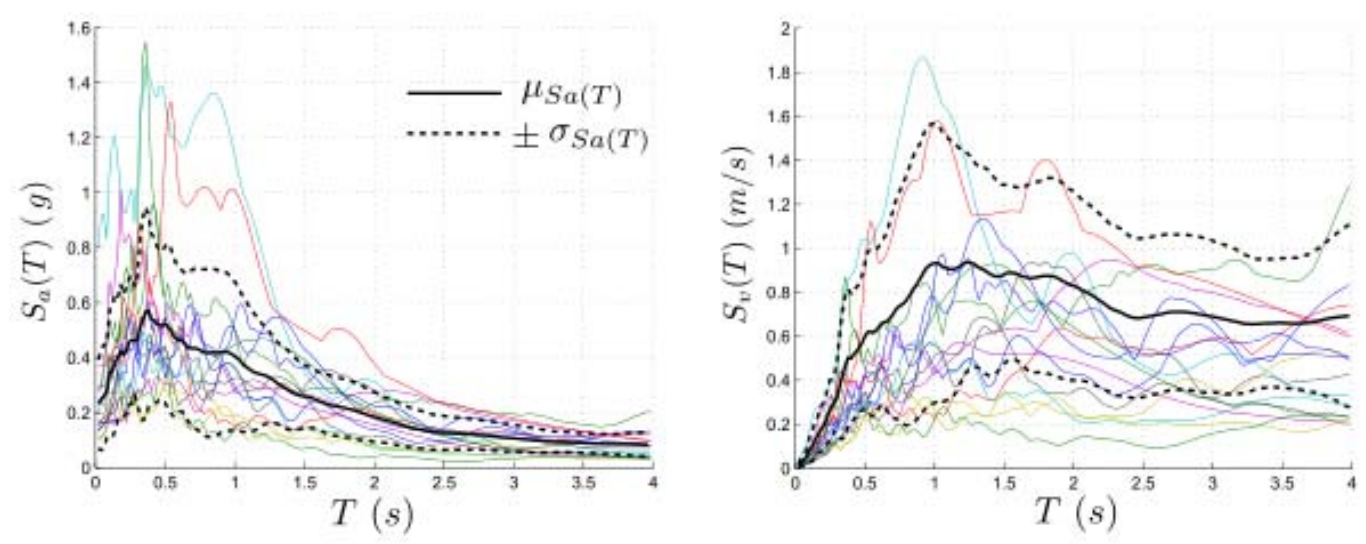

Fig. 1 - Elastic response spectra and the mean values 
The aspect mentioned above cant be surpassed by any of the ground-motion selection procedures. The aleatory nature of the ground-motions is not considered and generally the epistemic errors are not quantified by these types of methods. The result is that the response spectra will define an incomplete space of variables. Using a deterministic approach for selecting the response spectra will not guarantee that for some periods their associated spectral values will be conservative.

The entire set of used accelerograms should define a complete space of variables for the vector $I M$. This is a difficult task to achieve. Usually there are not enough accelerograms in the databases to define the complete space of variables. So the efficiency and sufficiency of the accelerograms are the only criteria for establishing if the chosen set of ground-motions should be used for computing the response spectra. There are two alternatives for generating response spectra: the uniform hazard spectra and the conditional spectra $[2,4]$. This two types of spectra are based on empirical ground-motion models [7], coupled with the probabilistic seismic analysis. The space of variables isn't restricted to the available data. The spectra choice is based on a period of exceedance and it guarantees that uniform hazard is associated to each oscillator.

\section{Inelastic response spectra of constant ductility}

The inelastic response spectrum is a representation of the absolute maximum response in EDP of an inelastic single degree of freedom system. Generally, the EDP is considered as the mean value plus a standard deviation obtained from a set of ground-motions. The EDP can be displacement, acceleration, velocity, dissipated hysteretic energy, number of plastic cycles or other parameter. In general quantities that are compared with the values obtained from an elastic spectrum are preferred, so that the ductility factor of the system $\mu$, the strength factor $q$ (behavior factor) or the inelastic displacement ratio $c$ is used.

The definition of the strength factor used in this paper is different from that given in the Romanian seismic design code. In the code, the strength factor is defined as the ratio of the maximum elastic lateral force to the yield strength of an elasto-perfect-plastic model. In the paper, the used system can have any cyclic law and the over-strength of the system has no influence in the definitions of the factors used herein.

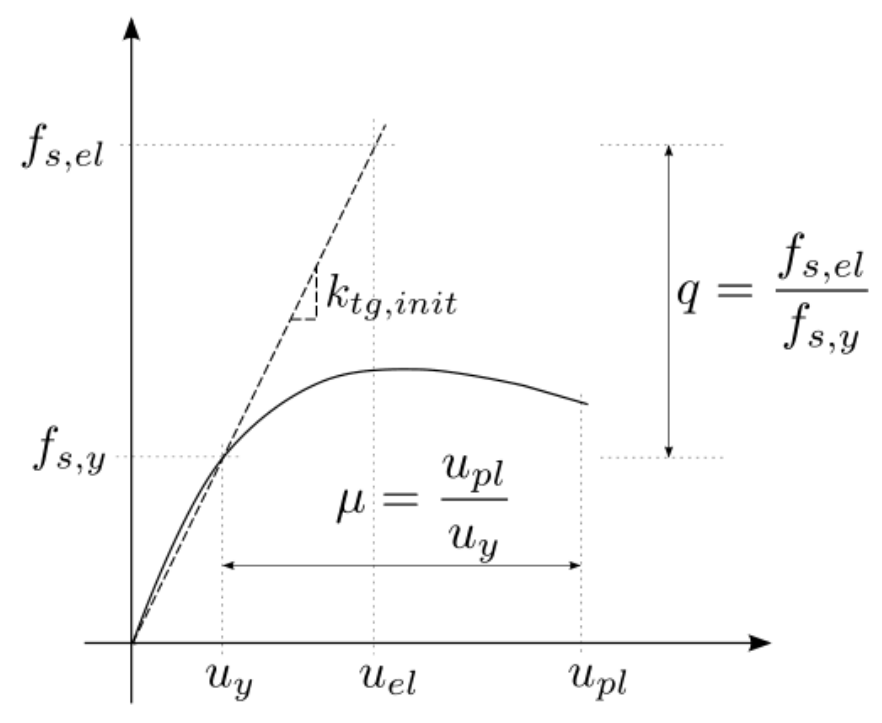

Fig. 2 - Equivalence between elastic and inelastic parameters [4]

Figure 2 presents the equivalence of the factors used herein, where $u_{y}$ is the displacement corresponding to the yield force, $u_{p l}$ is the maximum computed inelastic displacement and $u_{e l}$ is 
the elastic displacement, which is proportional to the maximum force $f_{s, e l}$ for the initial tangent stiffness of the inelastic single of degree system.

The characteristic yield force of the single degree of freedom system is computed by dividing the equivalent maximum elastic force to a strength factor $q$ chosen or computed for a specified ductility factor $\mu$. The inelastic displacement ratio is defined as the ratio of the plastic displacement to the elastic displacement and it can be written as:

$$
c=\frac{u_{p l}}{u_{e l}}=\frac{\mu}{q}
$$

In the Romanian code, the inelastic displacement ratio has a similar definition to that of equation (1), though there are some differences. One of these differences is that the parameter used in the code is for systems with constant yield force and it is conditioned by the ductility factor, the period of the system and the corner period. In earlier versions of the code, the value of the inelastic displacement ratio was a function of the periods only and, in the same time, it was limited at a maximum value equal to 2 . The definition presented in equation (1) is applied for a single degree of freedom system and no limit is imposed on the inelastic displacement ratio.

Five cyclic models of the material are selected for the inelastic SDOF system behavior: (a) elasto-perfect-plastic EPP, (b) elasto-plastic with hardening EPC, (c) Menegotto Pinto modified by Filippou $M P F$, (d) with loss of stiffness and strength $H 1$ and (e) with loss of strength only $H 2$. For each of these models, an initial tangent stiffness can be assigned using the maximum yield force and elastic displacement. The damping is proportional with the mass of the SDOF system and the value of the damping factor is $\xi=0.05$.

In order to compute inelastic spectra of constant ductility for a particular period $T_{i}$, the following relation between the ductility factor and the strength factor of the system can be assumed,

$$
q=\exp ^{a} \mu^{b}
$$

where the coefficients $a$ and $b$ are estimated using linear regression, considering that $\mu$ and $q$ are exponentially distributed. So, for a chosen value of the ductility factor, a strength factor is estimated for $T_{i}$ and the analysis of the nonlinear model is made with this estimated value of $q$. A procedure that follows the algorithm presented in $[5,6]$, including the equation (2), was implemented in OpenSees [10].
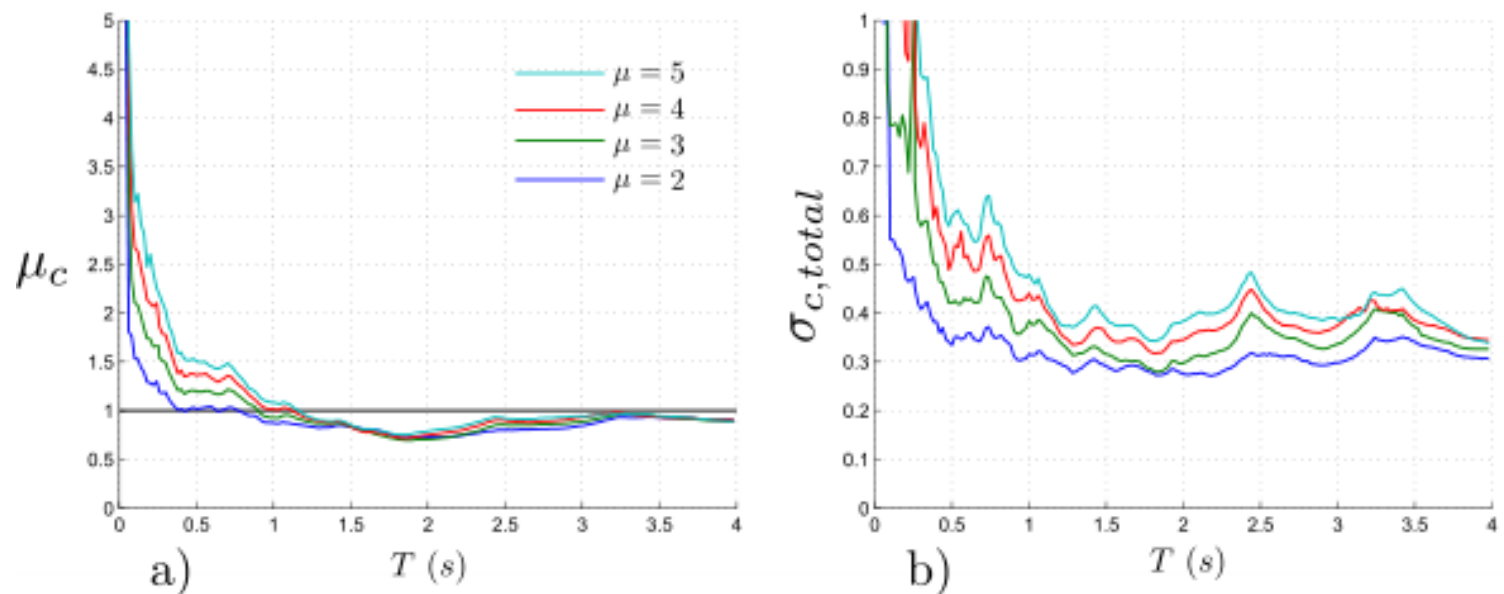

Fig. 3 - Inelastic spectra of constant ductility a) Mean of the inelastic displacement ratio $c$; b) Standard deviation.

Considering four values for the ductility factor and the response for all the cyclic laws, the spectra from figure 3 are plotted. For each ductility factor, a total number of 75 nonlinear analyses are 
done. The general conclusion is that for the short period oscillators, the inelastic displacement ratio is greater than 1 and for the long period range the factor is smaller or equal to 1 . The standard deviation is observed for all periods, with greater values for the short period interval.

The constant ductility spectra approximates the values of the response quantities for a fixed chosen ductility factor. In order to estimate these values an iterative solution is used because, by using equation (2), different values of the strength factor will be obtained. The initial step for computing the inelastic response spectra is to choose a ductility factor and, based on equation (2), to evaluate a strength factor. After the analysis is made, the obtained ductility factor is compared with the one chosen in the initial step. Generally, the initial $q$ factor has a different value from the solution obtained by iteration.

It is mentioned that equation (2) is not satisfied for all periods. For example, imposing a tolerance of 0.01 for the computed ductility factor, it was observed that for periods smaller than $0.05 \mathrm{~s}$, the error in the results varies from 1 to $100 \%$, and for periods greater than $0.05 \mathrm{~s}$, the error varies from 1 to $7 \%$. It is expected that the imposed ductility factor to be not correlated to the estimated strength factor for all periods. For periods that are smaller than $0.05 \mathrm{~s}$, if a nonlinear behavior is expected, the plastic demands can be much larger than for greater periods. In this cases, the authors recommend nonlinear behavior to be avoided.

The values estimated for the inelastic displacement ratio for the chosen ductility factors are plotted in figure 4 for two periods. The period from figure 4 (a) corresponds to the short period interval, while the period from figure 4 (b) corresponds to the long or medium period interval. It is observed the increasing of the inelastic displacement ratio $c$ with the increasing of the ductility factor.

The variability of $c$ is larger for systems with short periods, especially for high ductility factors. For systems with long periods, the variability is still present, but it is reduced for high ductility factors. For example, at periods of $0.20 \mathrm{~s}$ and ductility factor equal to 5 , the estimated inelastic displacement ratio, for some particular systems, can take values as high as 7...9. So for systems with periods in the short period interval, the use of high values for the strength factor or ductility factor will have an associated high degree of uncertainty in the estimated seismic response.

The response is affected by the frequency content of the ground-motion and the cyclic law of the material. These are two types of uncertainties in the model. The first one, which is a function of the number of used data, can be considered as an aleatory one, and the second one is function of the used model and of the influence of the frequency content. The total standard deviation presented in figure 3 (b) is not the sum of these two types of uncertainty, because these types have common elements.
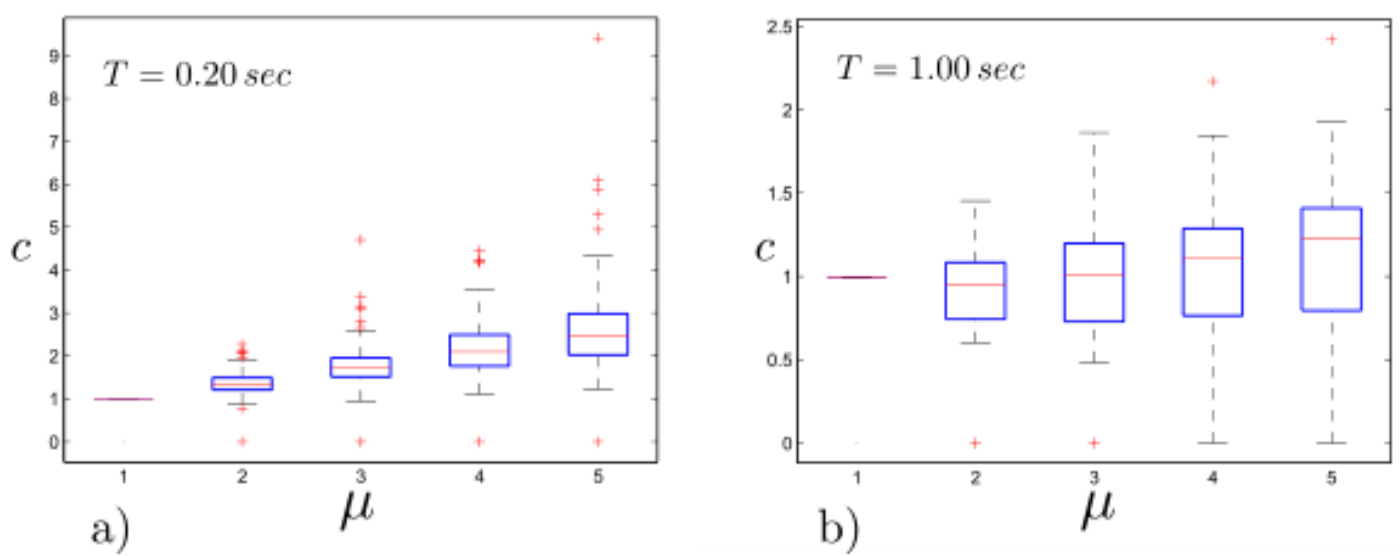

Fig. 4 - Influence of the ductility factor and period on the inelastic displacement ratio. 

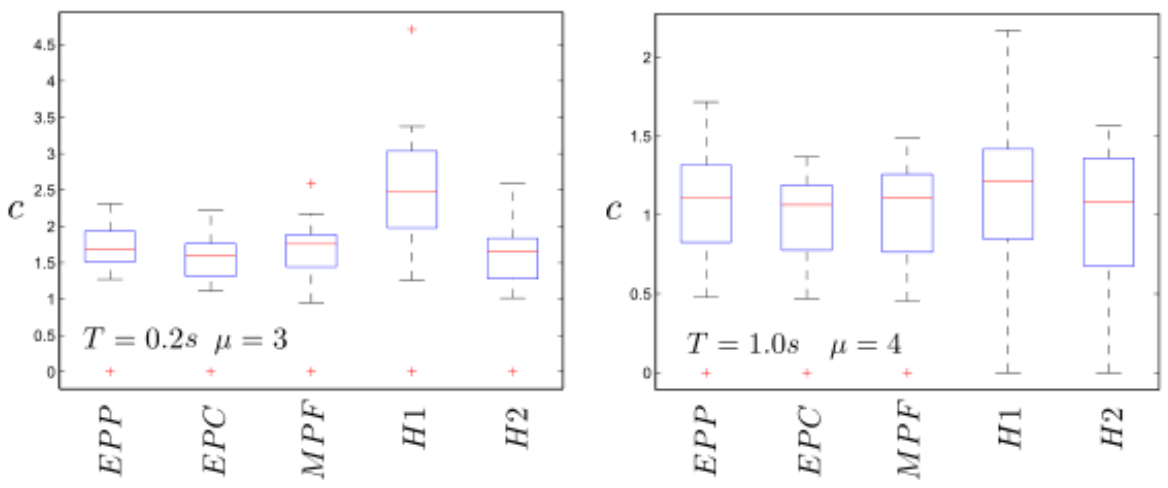

Fig. 5 - Influence of the hysteretic model on the inelastic displacement factor $C$

The influence of the hysteretic model is represented in figure 5. It can be observed that the highest values of the response are obtained for the system with stiffness and strength degradation. These differences are higher for the short period interval and are observed for all periods and chosen ductility factors. Another observation is that the variability of the response tends to be higher as the ductility factor is increased and the highest variability is associated with the $H 1$ model, with loss of strength and stiffness.

The model uncertainties influence on the response can be estimated using data for a single event and normalized at the fundamental period $T_{p}$. In figure 6 it is observed that the standard deviation of the response is correlated with $T_{p}$. The variability in a single event is a function of the frequency content and the hysteretic model. In figure 6 (b) the data is interpolated for all ductility factors and it is observed that the standard deviation increases as the ductility factor increases.

The separation of uncertainties in model uncertainty and response uncertainty exposes the fact that the variability of response is a function of the parameters of the dynamic model. For oscillators with period smaller than $T_{p}$, the influence of the model parameters in the response can be substantial. This is of particular interest when residual displacements are estimated [9] or when systems with loss of strength or stiffness are analyzed.
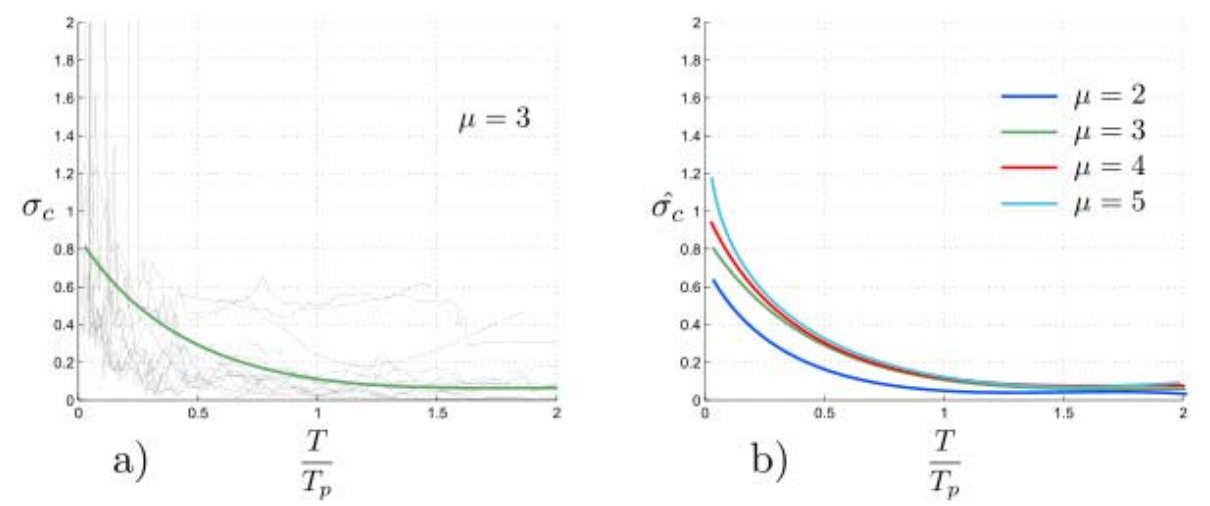

Fig. 6 - Variability in a single event for periods normalized at $T_{p}$ a) Standard deviation in a single event; b) interpolation on all data set

\section{Functional forms}

With data approximated using the inelastic response spectra, a functional form can be expressed. These functional forms are described using mathematical expressions for the relationship between the response parameters and those of the dynamic model and of the seismic action. It is proposed the functional form used in [12], having the following expression, 


$$
\hat{c}\left(T / T_{p}, \mu, \theta_{i}\right)=1+(\mu-1) \frac{1}{\theta_{1}\left(\frac{T}{T_{p}}\right)^{2}}+\theta_{2} \frac{T_{p}}{T} \exp \left[\theta_{3} \ln \left(\frac{T}{T_{p}}\right)^{2}\right]
$$

where $\theta_{i}$ are coefficients estimated by nonlinear regression on the available data [3]. Using the data corresponding to all hysteretic models and all accelerograms, the coefficients from table 1 are given.

Table 1

Coefficients for the functional form $\hat{c}\left(T / T_{p}, \mu, \theta_{i}\right)$ for the entire data set

\begin{tabular}{|c|c|c|c|}
\hline$\theta_{i}$ & $\mu=3$ & $\mu=4$ & $\mu=5$ \\
\hline$\theta_{1}$ & 11.665 & 11.867 & 18.929 \\
\hline$\theta_{2}$ & -0.142 & -0.219 & -0.167 \\
\hline$\theta_{3}$ & -0.518 & -0.514 & -0.522 \\
\hline
\end{tabular}

The nonlinear regression is made on constant ductility planes and the functional forms describe an approximate mean $\hat{c}$. The values are approximated for the complete interval of values. The correlation between the model parameters and the response vector EDP is ignored. The estimated values for $\hat{c}$ are not the same to those from figure 4 (a). Considering only the mean values of the inelastic displacement ratio, the coefficients from table 2 are estimated. These mean values are obtained by taking into account all hysteretic models.

Table 2

Coefficients for the functional form $\hat{c}\left(T / T_{p}, \mu, \theta_{i}\right)$ for mean values of hysteretic models

\begin{tabular}{|r|r|r|r|r|}
\hline$\theta_{i}$ & $\mu=2$ & $\mu=3$ & $\mu=4$ & $\mu=5$ \\
\hline$\theta_{1}$ & 630.08 & 13.212 & 25.017 & 19.425 \\
\hline$\theta_{2}$ & -0.100 & -0.125 & -0.095 & -0.166 \\
\hline$\theta_{3}$ & 0.850 & -0.518 & -0.522 & -0.521 \\
\hline
\end{tabular}

The statistic measure of how close the functional form is to the available data is denoted as the $\mathrm{r}^{2}$ coefficient or the determination coefficient. The $\mathrm{r}^{2}$ values obtained for the data sets are in general less than 0.50 . Usually a functional form is considered to approximate well the given data if $\mathrm{r}^{2}$ is greater then 0.50 and almost equal to 1.00. The obtained low values were expected given the strong variability in response observed in the previous section. The values of $r^{2}$ are increasing as the ductility factor is increased and have lowest values for the short period interval. Equation (3) identifies which oscillators can present consistent variability in the response as a function of the fundamental period.
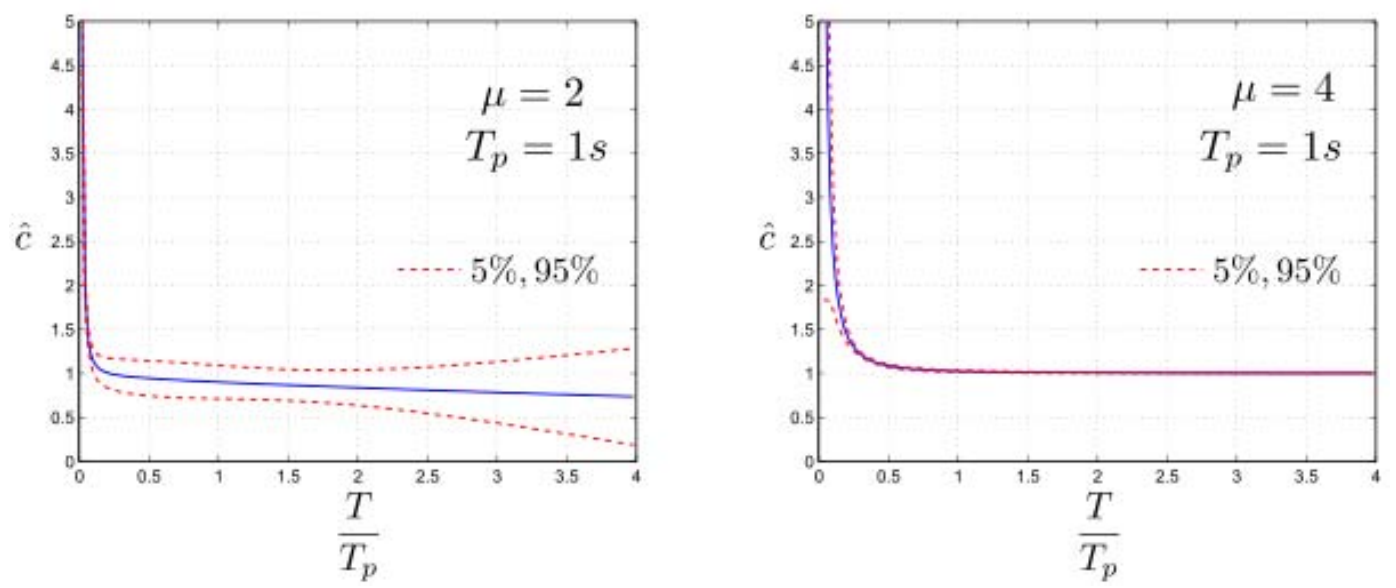

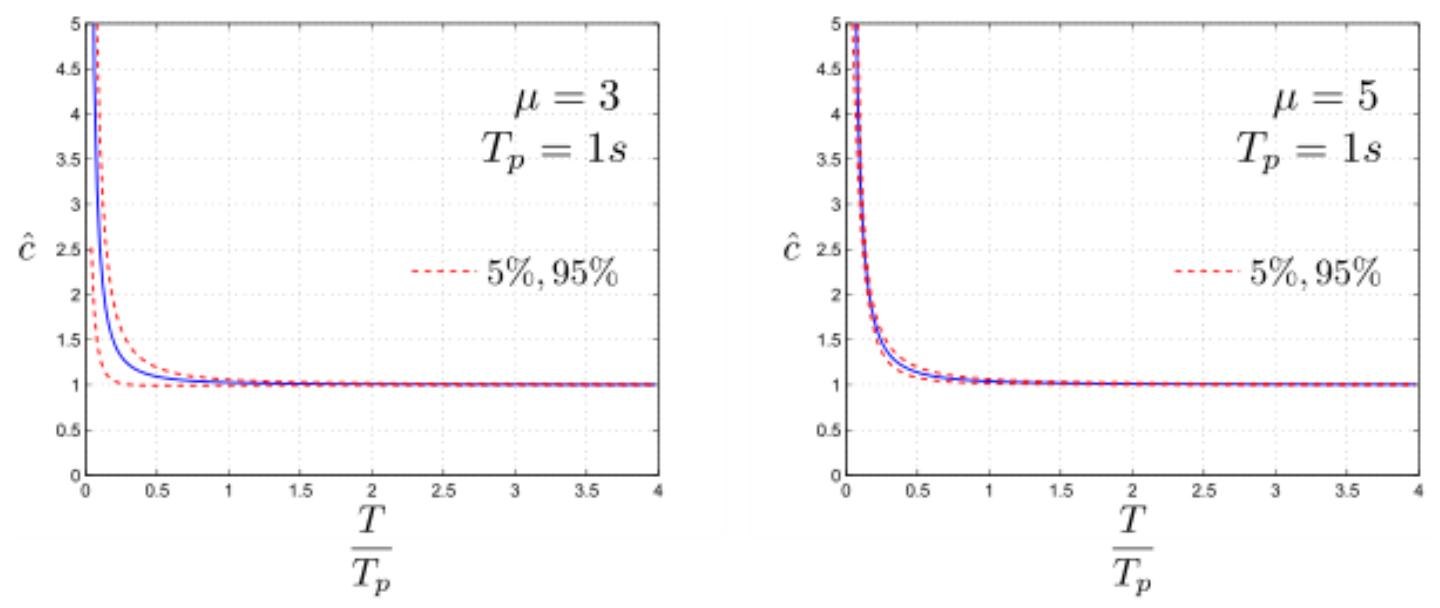

Fig. 7 - Response spectra of approximated mean $\hat{C}$ for different ductility factors using coefficients from table 2.

In figure 7, the response spectra of the mean values using equation (3) and the coefficients from table 2 are plotted. It can be observed that for the ductility factor equal to 2 , the proposed equation doesn't estimate with confidence the mean value $\hat{c}$ for all periods. For all other used ductility factors, the values approximated are reasonable for intervals greater than $T_{p}$. On the short period interval, that is for periods smaller than $T_{p}$, the mean keeps the observed variability in response.

Equation (3) keeps the observed particularities of the variability of response as in the previous section. As it was observed, the standard deviation of the response was present for all ductility factors. This standard deviation can be considered aleatory, ignoring its mixed nature. The data used in nonlinear regression in this case is that associated with the standard deviation observed for the mean values of the hysteretic models. To associate the uncertainties to equation (3), a stochastic term is added,

$$
\bar{c}\left(T / T_{p}, \mu, \theta_{i}, \beta_{i}\right)=\hat{c}\left(T / T_{p}, \mu, \theta_{i}\right)+\varepsilon_{\bar{c}}
$$

where $\varepsilon_{\bar{c}}$ is the stochastic component of $\bar{c}$. All the uncertainties are treated as aleatory with distribution $\varepsilon_{c}$, with zero mean and standard deviation $\sigma_{\varepsilon_{c}}=f\left(T / T_{p}, \mu, \beta_{i}\right)$.

Coefficients $\beta_{i}$ are approximated using nonlinear regression with a model that describes this standard deviation. The distribution of the stochastic term is considered log-normal and dependent on the variables used to estimate the mean $\hat{c}$.

The following functional form is proposed for approximating the standard deviation:

$$
\sigma_{\varepsilon_{c}}\left(T / T_{p}, q, \beta_{i}\right)=\frac{\mu-1}{\beta_{1}+\beta_{2}\left(\frac{T}{T_{p}}\right)+\left(\frac{T}{T_{p}}\right)^{\beta_{3}}}
$$

The data used is that of the standard deviation obtained for the mean values of $c$ from the hysteretic models. Because the data is considered with log-normal distribution, the following transformations are done: first, a transformation in log-format with the corresponding mean and standard deviation and, second, a backward transformation to normal variables. The final obtained coefficients using the nonlinear regression on the normal data are given in table 3 . 
Coefficients for the functional form $\sigma_{\varepsilon_{c}}\left(T / T_{p}, q, \beta_{i}\right)$

Table 3

\begin{tabular}{|r|r|r|r|}
\hline$\beta_{i}$ & $\mu=3$ & $\mu=4$ & $\mu=5$ \\
\hline$\beta_{1}$ & 0.0034 & -0.0087 & 0.0022 \\
\hline$\beta_{2}$ & 1.8777 & 5.7624 & 4.4652 \\
\hline$\beta_{3}$ & 1.4188 & 1.3649 & 1.3978 \\
\hline
\end{tabular}

The approximated values for the standard deviation of the regression model $\bar{c}$ are not equal to that of the available data. This standard deviation can be considered as specific for a period $T / T_{p}$.

In figure 8 the approximated values of the standard deviation for different ductility factors are plotted using coefficients from table 3 and equation 5. The $\mathrm{r}^{2}$ values are less than 0.50 and smaller than those obtained for the mean values. The variability of standard deviation is more pronounced and, for periods smaller than $T_{p}$, the values approximated keep a high degree of uncertainty.

Using the above equations, one can approximate the values for the inelastic displacement ratio $C$ and consider its intrinsic uncertainty for a specific period ratio $T / T_{p}$ and a chosen ductility factor. These equations approximate in a marginal way the values of $c$, the real function of $c$ being a strong nonlinear function. For some periods there is no correlation between ductility, period and $c$. The functional forms used herein have a few degrees of freedom, but its advantage is that it keeps the characteristics observed for the available data.

These functional forms are useful for choosing a yield level for a particular chosen ductility factor. They can be used in design when a displacement based design procedure is performed. For the assessment of structures, when the strength factor is known, using response spectra of constant ductility can lead to nonconservative results [12]. In this case, using data from the constant yield response spectrum, with a chosen strength factor, can lead to conservative estimates.

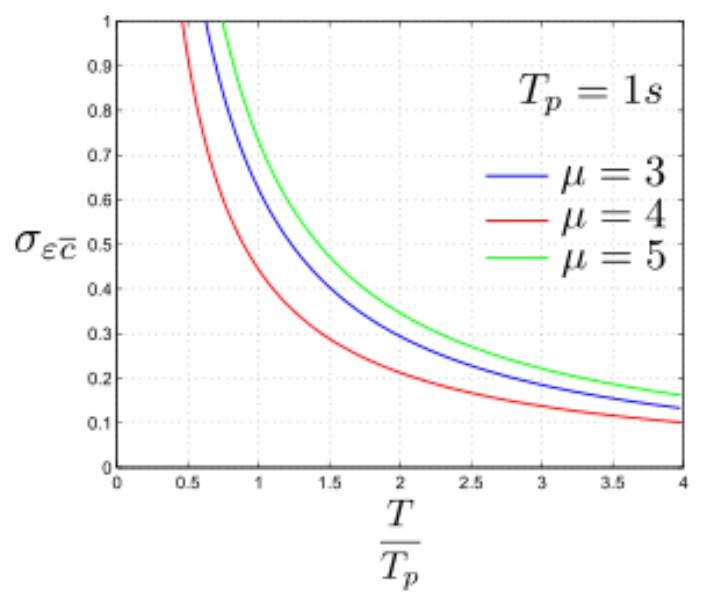

Fig. 8 - Approximated standard deviation using equation (5).

\section{Conclusions}

In the preceding sections the following procedures were exemplified:

- A ground-motion selection procedure that has two steps, in which accelerograms with similar frequency content to the ones picked for comparison are chosen. By using this procedure, real records are selected, that are neither scaled nor transformed.

- Use of inelastic response spectra of constant ductility for determining the inelastic displacement ratio and the influence of the hysteretic material model on the single degree of 
freedom response. It has been shown that for systems with loss of strength and stiffness the response is strongly affected. Using bilinear models for these systems is nonconservative.

- Choice of functional forms for approximating the inelastic displacement ratio as a function of SDOF period, fundamental period on site and ductility factor. These equations are approximate ones and can be used for evaluating the response of existing buildings.

The functional form presented herein estimates a mean and standard deviation for the inelastic displacement ratio. Considering that the probability distribution of $c$ is log-normal, with the approximated mean and standard deviation, estimates for the cumulative distribution functions are available. With these distributions and data from PSHA, a probabilistic seismic demand analysis for a particular oscillator can be done and the probability of exceedance for the inelastic displacement ratio can be approximated. These probabilities of exceedance are useful in performance based design.

The coefficients given in this paper for approximating inelastic displacement ratios are only for sites that are similar to Vrancea seismic zone. The used accelerograms have similar frequency content to the ones recorded at INCERC station at the seismic for the events from march 1977 and august 1986. They correspond for a soft soil site and with the fundamental period corresponding to all intervals. The functional form used herein is similar to other forms found in literature.

\section{References}

[1] Ancheta, T. D., Darragh, R. B., Stewart, J. P., Seyhan, E., Silva, W. J., Chiou, B. S. J., Wooddell, K. E., Graves, R. W., Kottke, A. R., Boore, D. M., Kishida, T. \& Donahue, J. L. (2013). PEER NGA-West2 Database. Berkeley: PEER. (Report No. 2013/03)

[2] Baker, J. W. (2011). Conditional Mean Spectrum: Tool for ground motion selection. Journal of Structural Engineering 137(3), 322-331. DOI: 10.1061/(ASCE)ST.1943-541X.0000215

[3] Bates, D. M. \& Watts, D. G. (2007). Nonlinear Regression Analysis and Its Applications. New York: JOHN WILEY \& SONS, INC.

[4] Bradley, B. A. (2012). A Ground Motion Selection Algorithm Based on the Generalized Conditional Intensity Measure Approach. Soil Dynamics and Earthquake Engineering 40,48-61. DOI: 10.1016/j.soildyn.2012.04.007

[5] Carr, A. J. (2003). The generation of inelastic response spectra for earthquake acceleration records. 7th Pacific Conference on Earthquake Engineering, 13 February 2003 (076). Christchurch, New Zealand.

[6] Chopra, A. K. (2007). Dynamics of Structures Theory and Applications to Earthquake Engineering, Third Edition. New Jersey: Prentice Hall.

[7] Douglas, J. \& Aochi, H. (2008). A survey of techniques for predicting earthquake ground motions for engineering purposes. Survey in Geophysics 29(1), 187-220. DOI: 10.1007/s10712-008-9046-y.

[8] Iervolino, I., Maddaloni, G. \& Cosenza, E. (2008). Eurocode 8 compliant real record sets for seismic analysis of structures. Journal of Earthquake Engineering 12(1), 54-90. 2008. DOI: 10.1080/13632460701457173.

[9] Liossatou, E. \& Fardis, M. N. (2014). Residual displacements of RC structures as SDOF systems. Earthquake Engineering \& Structural Dynamics 44(1), 713-734. DOI: 10.1002/eqe.2483

[10] McKenna, F., Fenves, G. L., Scott, M. H. \& Mazzoni, S. (2006). Open System for Earthquake Engineering Simulation [computer software]. Berkeley: PEER.

[11] MDRAP (2013). P100-1/2013 Cod de Proiectare Seismică. Partea I: Prevederi de Proiectare pentru Clădiri. România

[12] Ruiz-Garcia, J. \& Miranda, E. (2005). Performance-Based Assessment of Existing Structures Accounting for Residual Displacements. Stanford University: John A. Blume Earthquake Engineering Center. (Report No. 153)

[13] Shahi, S. K. \& Baker, J. W. (2011). An empirically calibrated framework for including the effects of near-fault directivity in Probabilistic Seismic Hazard Analysis. Bulletin of the Seismological Society of America 101(2), 742-755. DOI: 10.1785/0120100090.

[14] Shome, N. \& Cornell, C. A. (1999). Probabilistic seismic demand analysis of nonlinear structures. Stanford University: John A. Blume Earthquake Engineering Center. (Report No. RMS-35)

[15]Zelaschi, C., Monteiro, R., Marques, M. \& Pinho, R. (2014). Comparative analysis of intensity measures for reinforced concrete bridges. Second European Conference on Earthquake Engineering, 25-29 august 2014. Istanbul, Turkey. 


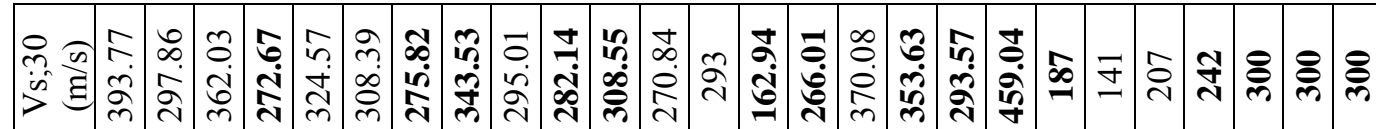

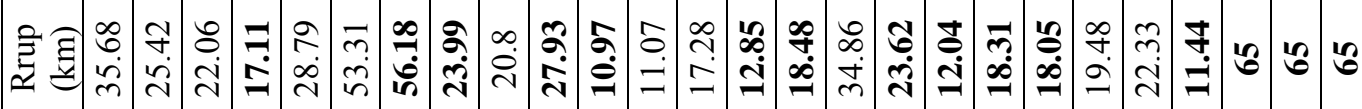

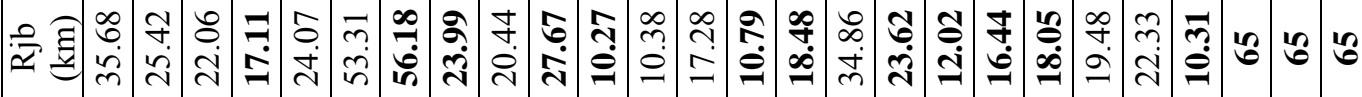

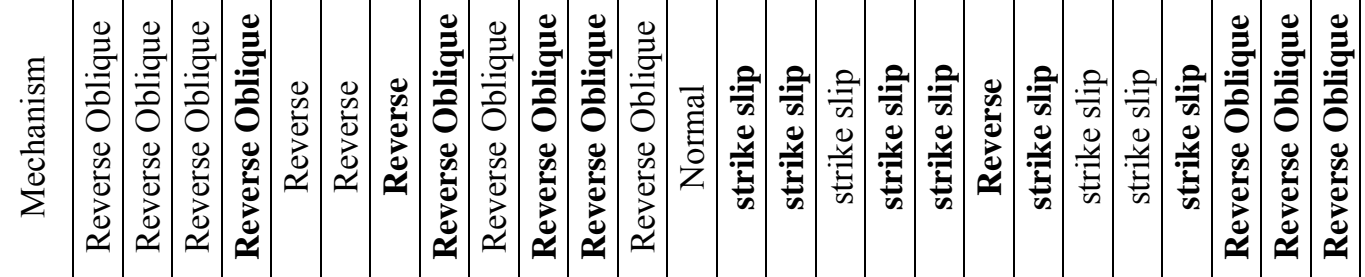

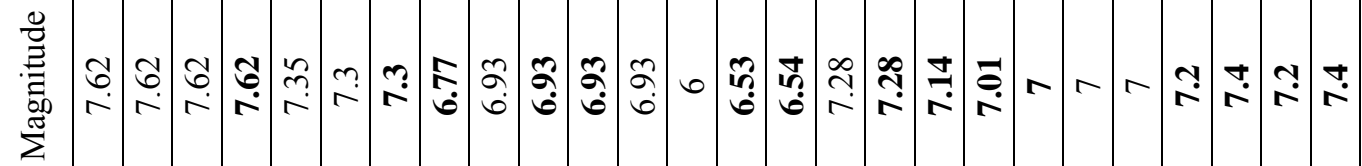

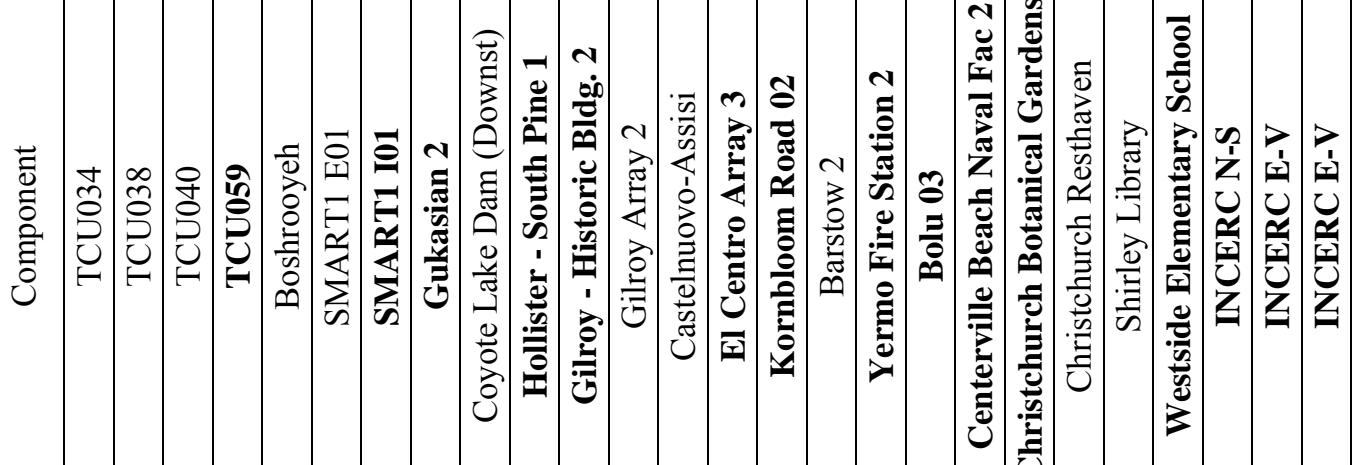

売

离
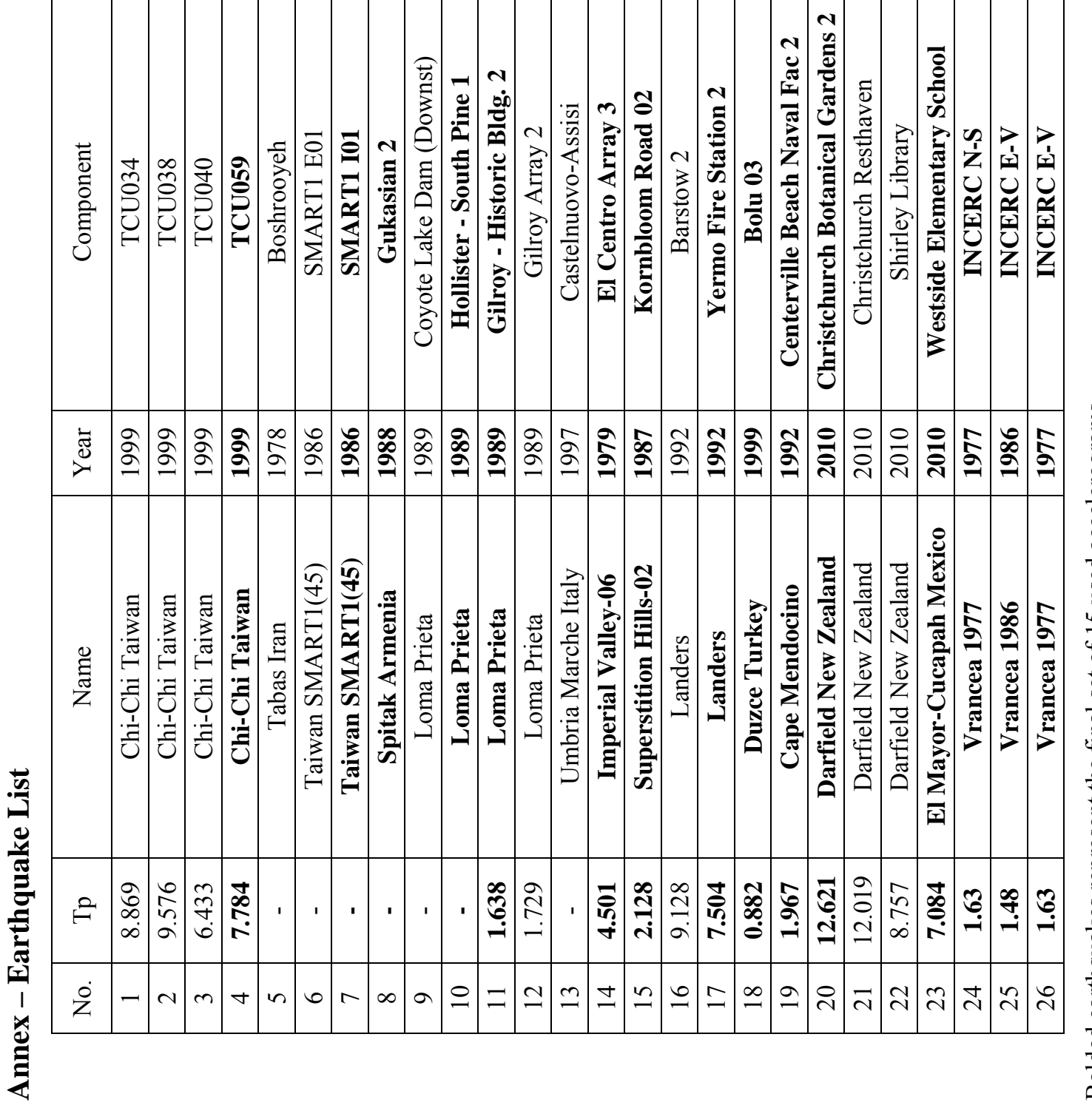